Research Article

\title{
Critical Parameters and Influence on Dynamic Behaviours of Nonlinear Electrostatic Force in a Micromechanical Vibrating Gyroscope
}

\author{
Shuying Hao $\mathbb{D},{ }^{1,2}$ Yulun Zhu, ${ }^{1,2}$ Yuhao Song, ${ }^{1,2}$ Qichang Zhang, ${ }^{3}$ Jingjing Feng, ${ }^{1,2}$

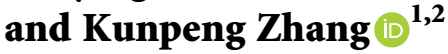 \\ ${ }^{1}$ Tianjin Key Laboratory for Advanced Mechatronic System Design and Intelligent Control, School of Mechanical Engineering, \\ Tianjin University of Technology, Tianjin 300384, China \\ ${ }^{2}$ National Experimental Teaching Demonstration Center of Mechanical and Electrical Engineering, \\ Tianjin University of Technology, Tianjin 300384, China \\ ${ }^{3}$ Tianjin Key Laboratory of Nonlinear Dynamics and Control, Department of Mechanics, School of Mechanical Engineering, \\ Tianjin University, Tianjin 300372, China
}

Correspondence should be addressed to Kunpeng Zhang; kpzhang@email.tjut.edu.cn

Received 3 April 2020; Revised 27 September 2020; Accepted 14 October 2020; Published 27 October 2020

Academic Editor: Marco Alfano

Copyright (c) 2020 Shuying Hao et al. This is an open access article distributed under the Creative Commons Attribution License, which permits unrestricted use, distribution, and reproduction in any medium, provided the original work is properly cited.

The electrostatic force nonlinearity caused by fringe effects of the microscale comb will affect the dynamic performance of the micromechanical vibrating gyroscopes (MVGs). In order to reveal the influence mechanism, a class of four-degree-of-freedom (4DOF) electrostatically driven MVG is considered. The influence of DC bias voltage and comb spacing on the nonlinearity of electrostatic force and the dynamic response of the MVG by using multiple time scales method and numerical simulation are discussed. The results indicate that the electrostatic force nonlinearity causes the system to show stiffness softening. The softening characteristics of the electrostatic force cause the offset of the resonance frequency and a decrease in sensitivity. Although the electrostatic nonlinearity has a great influence on the dynamic behaviour, its influence can be avoided by the reasonable design of the comb spacing and DC bias voltage. There exists a critical value for comb spacing and DC bias voltage. In this paper, determining the critical values is demonstrated by nonlinear dynamics analysis. The results can be supported by the finite element analysis and numerical simulation.

\section{Introduction}

Micromechanical gyroscopes are a kind of inertial sensors used to measure the angular velocity or angular displacement. The first micromechanical gyroscope with doubleframe structure was designed by American Draper Labs in 1988 [1], and then the different kinds of micromechanical gyroscopes emerged gradually. Compared with the traditional gyroscopes, micromechanical gyroscopes have many advantages such as low cost, light weight, small size, mass production, digitization, and intelligence. For all classes of micromechanical gyroscopes, the micromechanical vibrating gyroscopes (MVGs) are most widely applied in many fields, including consumer electronics, automotive stabilization, navigation and guidance, robotics applications, and virtual reality [2].

The working principle of MVGs is based on energy transfer among the drive mode and the sense mode via Coriolis effect. The drive mode is usually employed by the electrostatic actuation mechanism [3]. Numerous studies have shown that there are many nonlinear factors in the MVG system, involving the most common stiffness nonlinearity for the large deformation of the supporting microbeam and electrostatic force nonlinearity for fringe effect of the microcomb. These factors will cause frequency offset, multistable solution, softening-hardening characteristics, 
and transition of stiffness in the MVGs $[4,5]$. In fact, both the softening and hardening characteristics would be accompanied by the jump phenomenon. It means that the routes of vibration amplitude are different with the sweepup and sweep-down of the excitation frequency. A more detailed discussion can be found in $[6,7]$.

The hardening characteristics of stiffness nonlinearity become significant when the driven vibration amplitude increases. It also extremely affects the dynamic performance of MVGs. Many studies focused on the nonlinear dynamics of MVGs with considering the stiffness nonlinearity. Tsai and Sue [8] researched the nonlinear dynamics of a kind of wheel micro gyroscope. Chaotic behaviours were analysed by using bifurcation diagrams. It was verified that the transition behaviours of different motion modes of drive mode and sense mode had a similar orbit. Kenig et al. [9] studied a pair of parametrically driven gyroscopes with coupling nonlinearity, the existence of homoclinic orbit was confirmed by analytical methods, and the existence of chaos was proved by the generated Silnikov orbit. Tatar et al. [10] successfully linearized the electrostatic nonlinearity at the driving comb using a formed comb with a tuned cubic hardening compensation in a triple symmetric silicon-oninsulator (SOI) MEMS micro gyroscope. This tuning method not only achieves high driving displacement but also keeps the phase frequency response under linear condition and has better bias instability. Lajimi et al. [11] studied the influence of DC bias voltage, AC drive voltage, driving frequency, and quality factors on the response of rigid beam micro gyroscope system. The study showed that the micro gyroscope has larger bandwidth and higher sensitivity when it works in the nonlinear region. Hao et al. $[12,13]$ analysed the influence of driving stiffness and detection stiffness with cubic nonlinearity on the dynamic performance of single drive mode and double sense mode micro gyroscope, respectively. The studies showed that, with the increase of stiffness nonlinearity, the driving and sensing of micro gyroscope had complex nonlinear behaviours and lead to serious instability of the micro gyroscope system.

The above research is mainly focused on the stiffness nonlinearity of MVGs. It is worth mentioning that stiffness nonlinearity is usually caused by the large deformation of the straight beam in the MVGs. However, the traditional straight beam has been replaced with the folded beam in the design of the new MVGs recently. Thus, the influence of the stiffness nonlinearity on the MVGs can be reduced gradually.

Generally, the electrostatic driving force of MVGs is designed by the requirements of the sensitivity according to the linear system. It is not usually considered that nonlinear characteristics of electrostatic force influence the MVG. However, electrostatic force nonlinearity can cause the softening characteristics of the output response of MVG, leading to a reduction of natural frequencies, jumping phenomena, and instability of sensitivity [14]. Chouvion et al. [15] investigated the influence of nonlinear electrostatic force on the dynamic characteristics of a capacitive vibrating ring gyroscope under strong impact. The results showed that the nonlinear electrostatic force could induce mode coupling, amplitude jump, and performance degradation of the sensor in the case of resonance. Liang et al. [16] studied the dynamic response of a ring vibrating gyroscope by using the method of linear and nonlinear numerical analysis. These results showed that the sensitivity of ring gyroscope is affected by the geometrical nonlinearity and the electrostatic force nonlinearity. Sieberer et al. [17] considered a ring resonator with 8 uniformly spaced support legs. When a severe in-plane shock is applied, the rigid body response of the ring reduces the electrode gap significantly and causes the electrostatic force nonlinearity.

The comb spacing and DC bias voltage have great influence on the nonlinearity of electrostatic force and the output performance of the MVG. Nonlinear electrostatic forces may cause the failure of the MVG based on the linear structural designs. Considering the determination of the minimum comb spacing and the maximum DC bias voltage, referred to herein as the critical value, become very important. However, there are few reports on the related research.

In the present work, a kind of four-degree-of-freedom (4-DOF) MVG with double drive mode and double sense mode was considered. Nonlinear dynamic equation of the MVG was solved by using the multiple time scales method. The influence of the electrostatic force nonlinearity on the dynamic performance was studied by the approximate analytical solution. The critical value of the driving comb spacing and DC bias voltage can be determined by the analysis of nonlinear dynamic characteristics. The reliability of analysis method and results was supported by the finite element method and numerical simulation.

\section{Working Principle of MVG with Double Drive Mode and Double Sense Mode}

A 4-DOF MVG with double drive mode and double sense mode [18] is considered in this paper, as illustrated in Figure 1. This kind of MVG is mainly composed of driving mass, decoupled mass, conversion mass, sensing mass, elastic microbeams, and comb electrodes.

The drive direction is along the $x$-axis and the sense direction is along the $y$-axis. $\Omega_{z}$ is the input angular velocity and is perpendicular to the $(x-y)$ plane. The decoupled mass $m_{f}$ and conversion mass $m_{2}$ compose a two-stage decoupled structure, which plays the role of isolation drive mode and sense mode. The driving mass $m_{1}$ oscillates in the $x$ direction under the action of the driving comb electrode. The decoupling mass $m_{f}$ begins to oscillate in $x$ direction due to the action of $k_{2}$. Meanwhile, the conversion mass $m_{2}$ oscillates along the $x$ direction with the decoupled mass $m_{f}$ under the action of $k_{4}$. Because of the Coriolis effect, the vibration in $x$ direction causes the resonance in $y$ direction when the system has angular velocity $\Omega_{z}$ input in the vertical direction of the $(x-y)$ plane. Then, the conversion mass $m_{2}$ and the detection mass $m_{3}$ oscillate in $y$ direction under the constraints of $k_{4}, k_{5}$, and $k_{6}$.

The displacement of $m_{3}$ in $y$ direction is the output displacement in the sense direction, which increases with the increase of $\Omega_{z}$. Because the output amplitude is directly 


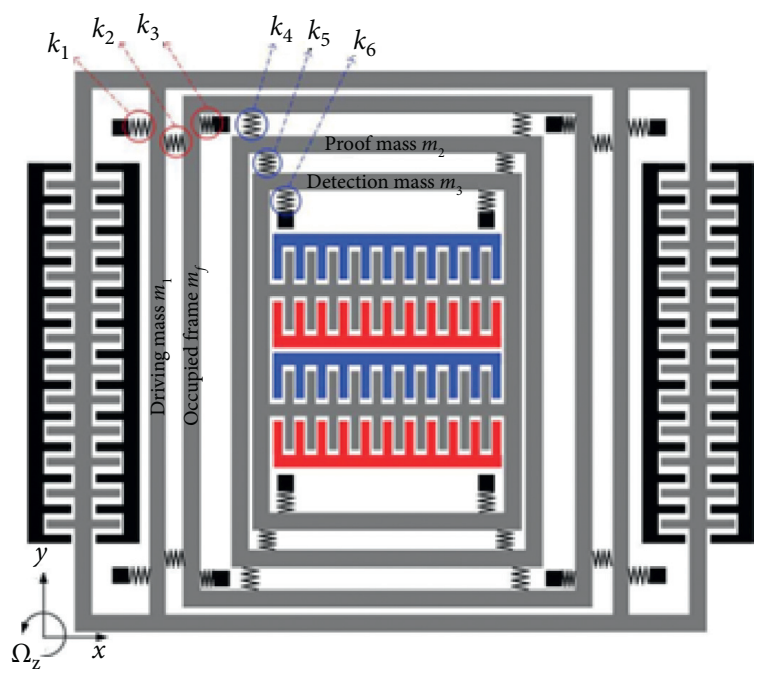

FIGURE 1: Schematic diagram of the MVG with double drive mode and double sense mode.

proportional to the input angular velocity $\Omega_{z}, \Omega_{z}$ of carrier can be obtained by measuring the output amplitude.

The dynamic model of a 4-DOF MVG is shown in Figure 2. Driving mass $m_{1}$ is defined as Drive-I, conversion mass $m_{2}$ and decoupled mass $m_{f}$ are combined to Drive-II, conversion mass $m_{2}$ is defined as Sense-I, and sense mass $m_{3}$ is defined as Sense-II.

Considering that MVG usually works in a vacuum environment, the air damping is relatively small. So the nonlinear factors of the damping are neglected. Therefore, it can be assumed that dampings in the drive direction and sense direction are both linear ones. The dynamic equations of the 4-DOF MVG are established, as shown in (1) and (2).

Drive direction is as follows:

$\left\{\begin{array}{l}m_{1} \ddot{x}_{1}+\left(c_{1}+c_{2}\right) \dot{x}_{1}-c_{2} \dot{x}_{2}+\left(k_{1}+k_{2}\right) x_{1}-k_{2} x_{2}=F_{\mathrm{d}}, \\ \left(m_{2}+m_{f}\right) \ddot{x}_{2}-c_{2} \dot{x}_{1}+\left(c_{2}+c_{3}\right) \dot{x}_{2}-k_{2} x_{1}+\left(k_{2}+k_{3}\right) x_{2}=0 .\end{array}\right.$

Sense direction is as follows:

$$
\left\{\begin{array}{l}
m_{2} \ddot{y}_{1}+\left(c_{4}+c_{5}\right) \dot{y}_{1}-c_{5} \dot{y}_{2}+\left(k_{4}+k_{5}\right) y_{1}-k_{5} y_{2}=F_{c}, \\
m_{3} \ddot{y}_{2}-c_{5} \dot{y}_{1}+\left(c_{5}+c_{6}\right) \dot{y}_{2}-k_{5} y_{1}+\left(k_{5}+k_{6}\right) y_{2}=0
\end{array}\right.
$$

where $x_{i}(i=1,2)$ is the displacement of the $i$-th DOF in the drive direction, $y_{i}(i=1,2)$ is the displacement of the $i$-th DOF in the sense direction, $m_{i}(i=1,2,3,4, f)$ are the driving mass, proof mass, sensing mass, and decoupled mass, respectively, $c_{i}(i=1,2,3,4,5,6)$ is damping coefficient, $k_{i}(i=1,2,3,4,5,6)$ is the equivalent stiffness coefficient of each elastic microbeam, and $F_{d}$ and $F_{c}$ are electrostatic driving force and Coriolis force, respectively. The sense mode is coupled to the drive mode by Coriolis force $\left(F_{c}=-2 m_{2} \Omega_{z} \dot{x}_{2}\right)$ into a 4 -DOF system.

The structure of a pair of electrostatic driving comb is shown in Figure 3, where $w$ is the width of the comb, $h$ is the thickness of the comb, $d$ is the clearance within comb space, $L$ is the length of the comb, and $l_{0}$ is the initial overlap length. $g_{0}$ is the distance between the end face of the movable comb and the bottom face of the fixed comb $\left(g_{0}=L-l_{0}\right)$.

Considering the fringe effects [19], the total electrostatic force $F_{d}$ of the variable area capacitor is expressed as follows:

$$
F_{d}=V_{d}^{2}\left[\frac{P_{1}}{\left(g_{0}-x\right)^{2}}-\frac{P_{1}}{\left(g_{0}+x\right)^{2}}\right]+4 P_{2} V_{d} V_{a} \cos \left(\omega_{0} t\right) .
$$
$x=0$.

Equation (3) is as follows through Taylor expansion at

$$
F_{d}=\frac{P_{1} V_{d}^{2}}{g_{0}^{2}}\left[4 \frac{x}{g_{0}}+8\left(\frac{x}{g_{0}}\right)^{3}\right]+4 P_{2} V_{d} V_{a} \cos \left(\omega_{0} t\right)+O\left(x^{5}\right)
$$

where $P_{1}=\left(n \varepsilon_{0} \varepsilon_{r} w h / 2\right), P_{2}=\left(n \varepsilon_{0} \varepsilon_{r} / \pi\right)\{1+\ln [1+(2 \pi h / d)$ $+\ln (1+(2 \pi h / d))]\}, n$ is the number of combs, $\varepsilon_{0}$ is the dielectric constant of the air, $\varepsilon_{r}$ is the relative permittivity, $V_{d}$ is the DC bias voltage, $V_{a}$ is the AC excitation voltage, and $\omega_{0}$ is the electrostatic drive frequency.

\section{Nonlinear Perturbation Analysis}

The stiffness softening caused by the electrostatic force nonlinearity will lead to the serious instability of the system. It has a serious impact on the sensitivity and bandwidth. In order to investigate the dynamic behaviour of the system, the electrostatic force nonlinearity between the driving comb teeth needs to be considered.

Substituting equation (4) into (1), the following can be obtained:

$$
\left\{\begin{array}{l}
m_{1} \ddot{x}_{1}+\left(c_{1}+c_{2}\right) \dot{x}_{1}-c_{2} \dot{x}_{2}+\left(k_{1}+k_{2}\right) x_{1}-k_{2} x_{2}=\frac{P_{1} V_{d}^{2}}{g_{0}^{2}}\left[4 \frac{x_{1}}{g_{0}}+8\left(\frac{x_{1}}{g_{0}}\right)^{3}\right]+4 P_{2} V_{d} V_{a} \cos \left(\omega_{0} t\right), \\
\left(m_{2}+m_{f}\right) \ddot{x}_{2}-c_{2} \dot{x}_{1}+\left(c_{2}+c_{3}\right) \dot{x}_{2}-k_{2} x_{1}+\left(k_{2}+k_{3}\right) x_{2}=0 .
\end{array}\right.
$$




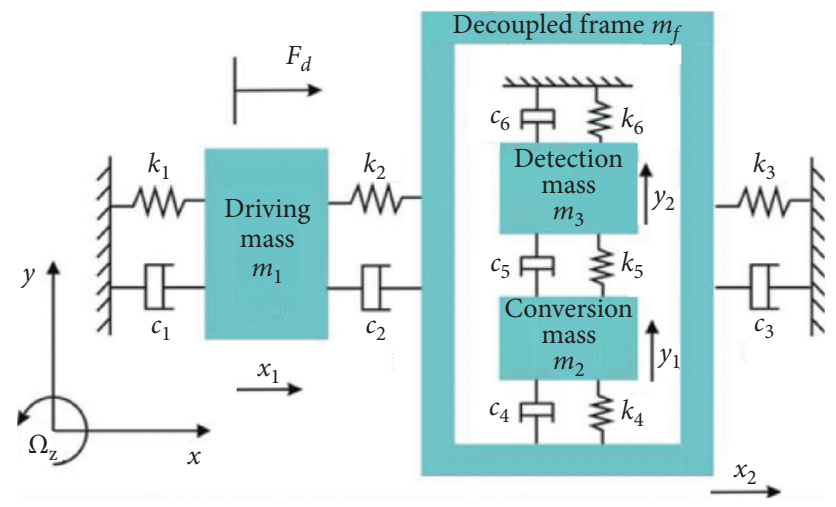

FIgURe 2: Dynamic model of the 4-DOF MVG.

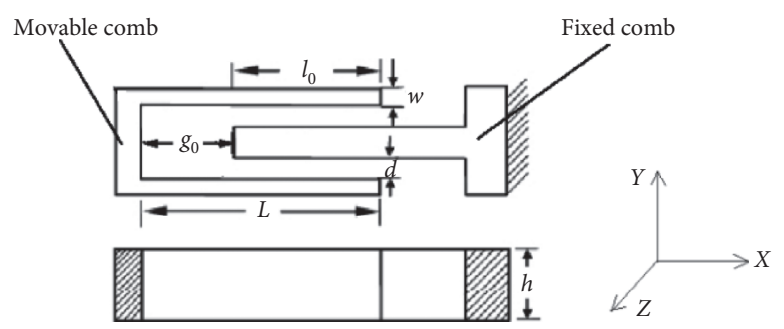

Figure 3: Schematic diagram of the comb structure.

Simplify equation (5) and obtain the dynamic equation:

$$
\left\{\begin{array}{l}
\ddot{x}_{1}+\alpha_{3} \dot{x}_{1}-\alpha_{4} \dot{x}_{2}+\left(\alpha_{1}-\frac{4 \gamma_{1} V_{d}^{2}}{g_{0}^{3}}\right) x_{1}-\alpha_{2} x_{2}-\frac{8 \gamma_{1} V_{d}^{2}}{g_{0}^{5}} x_{1}^{3} \\
\quad=4 \gamma_{2} V_{d} V_{a} \cos \left(\omega_{0} t\right), \\
\ddot{x}_{2}-\beta_{3} \dot{x}_{1}+\beta_{4} \dot{x}_{2}-\beta_{1} x_{1}+\beta_{2} x_{2}=0
\end{array}\right.
$$

where $\quad \alpha_{1}=\left(k_{1}+k_{2} / m_{1}\right), \quad \alpha_{2}=\left(k_{2} \alpha_{1} / m_{1}\right)$, $\alpha_{3}=\left(c_{1}+c_{2} / m_{1}\right), \quad \alpha_{4}=\left(c_{2} / m_{1}\right), \quad \beta_{1}=\left(k_{2} /\left(m_{2}+m_{f}\right)\right)$, $\beta_{2}=\left(k_{2}+k_{3} /\left(m_{2}+m_{f}\right)\right), \beta_{3}=\left(c_{2} /\left(m_{2}+m_{f}\right)\right), \beta_{4}=\left(c_{2}+\right.$ $\left.c_{3} /\left(m_{2}+m_{f}\right)\right)$, and $\gamma_{1}=\left(P_{1} / m_{1}\right), \gamma_{2}=\left(P_{2} / m_{1}\right)$.

Equation (6) can be regarded as the forced vibration of Duffing system with damping under simple harmonic excitation. The perturbation solution is obtained by multiple time scales method [20]. The first primary resonances with $1: 1$ internal resonance conditions $\left(\omega_{0}^{2}=\omega_{1}^{2}+\varepsilon^{2} \sigma_{1}\right.$, $\omega_{2}^{2}=\omega_{1}^{2}+\varepsilon^{2} \sigma_{2}$ ) are considered simultaneously, where $\omega_{1}$ and $\omega_{2}$ are the resonance frequencies of the first-order mode and second-order mode in the drive direction, respectively. $\mathcal{E}$ is introduced as a small parameter. Two detuning parameters $\sigma_{1}$ and $\sigma_{2}$ were introduced. The dynamic equation (7) can be modified as

$$
\left\{\begin{array}{l}
\ddot{x}_{1}+\omega_{0}^{2} x_{1}=-\varepsilon^{2}\left(\widehat{\alpha}_{1}-\frac{4 \widehat{\gamma}_{1} V_{d}^{2}}{g_{0}^{3}}\right) x_{1}+\varepsilon^{2} \widehat{\alpha}_{2} x_{2}-\varepsilon^{2} \widehat{\alpha}_{3} \dot{x}_{1}+\varepsilon^{2} \widehat{\alpha}_{4} \dot{x}_{2}+\frac{8 \widehat{\gamma}_{1} V_{d}^{2}}{g_{0}^{5}} x_{1}^{3} \\
+\varepsilon^{3} 4 \widehat{\gamma}_{2} V_{d} V_{a} \cos \left(\omega_{0} t\right)+\varepsilon^{2}\left(\widehat{\omega}_{1}^{2}+\sigma_{1}\right) x_{1}, \\
\ddot{x}_{2}+\omega_{0}^{2} x_{2}=\varepsilon^{2} \widehat{\beta}_{1} x_{1}-\varepsilon^{2} \widehat{\beta}_{2} x_{2}+\varepsilon^{2} \widehat{\beta}_{3} \dot{x}_{1}-\varepsilon^{2} \widehat{\beta}_{4} \dot{x}_{2}+\varepsilon^{2} \widehat{\omega}_{2}^{2} x_{2}+\varepsilon^{2}\left(\sigma_{1}-\sigma_{2}\right) x_{2},
\end{array}\right.
$$

where $\quad \alpha_{i}=\varepsilon^{2} \widehat{\alpha}_{i}(i=1,2,3,4), \quad \beta_{i}=\varepsilon^{2} \widehat{\beta}_{i}(i=1,2,3,4)$, $\omega_{i}^{2}=\varepsilon^{2} \widehat{\omega}_{i}^{2}(i=1,2)$, and $\gamma_{i}=\varepsilon^{2} \widehat{\gamma}_{i}(i=1,2)$.

The approximate solution of equation (7) can be written in the following form:

$$
\begin{aligned}
& x_{1}=\varepsilon x_{11}\left(T_{0}, T_{2}\right)+\varepsilon^{3} x_{13}\left(T_{0}, T_{2}\right)+\cdots, \\
& x_{2}=\varepsilon x_{21}\left(T_{0}, T_{2}\right)+\varepsilon^{3} x_{23}\left(T_{0}, T_{2}\right)+\cdots,
\end{aligned}
$$

where $T_{n}=\varepsilon^{n} t,(\mathrm{n}=1,2,3, \ldots)$. 
Substituting equation (8) into (7) and comparing the coefficients of the same order at both ends of the equation, the following partial differential equations can be obtained:

$$
\begin{aligned}
O\left(\varepsilon^{1}\right): D_{0}^{2} x_{11}+\omega_{0}^{2} x_{11}= & 0, \\
D_{0}^{2} x_{21}+\omega_{0}^{2} x_{21}= & 0, \\
O\left(\varepsilon^{3}\right): D_{0}^{2} x_{13}+\omega_{0}^{2} x_{13}= & -D_{0}\left(2 D_{2} x_{11}+\widehat{\alpha}_{3} x_{11}\right)-\left(\widehat{\alpha}_{1}-\frac{4 \widehat{\gamma}_{1} V_{d}^{2}}{g_{0}^{3}}\right) x_{11}+\widehat{\alpha}_{2} x_{21}+\widehat{\alpha}_{4} D_{0} x_{21} \\
& +\frac{8 \widehat{\gamma}_{1} V_{d}^{2}}{g_{0}^{5}} x_{11}^{3}+4 \widehat{\gamma}_{2} V_{d} V_{a} \cos \left(\omega_{0} t\right)+\left(\widehat{\omega}_{1}^{2}+\sigma_{1}\right) x_{11}, \\
D_{0}^{2} x_{23}+\omega_{0}^{2} x_{23}= & -D_{0}\left(2 D_{2} x_{21}+\widehat{\beta}_{4} x_{21}\right)+\widehat{\beta}_{1} x_{11}-\widehat{\beta}_{2} x_{21}+\widehat{\beta}_{3} D_{0} x_{11}+\widehat{\omega}_{2}^{2} x_{21}+\left(\sigma_{1}-\sigma_{2}\right) x_{21} .
\end{aligned}
$$

The general solution form of equation (9) can be expressed as follows:

$x_{11}\left(T_{0}, T_{2}\right)=A_{11}\left(T_{2}\right) \exp \left(i \omega_{0} T_{0}\right)+\bar{A}_{11}\left(T_{2}\right) \exp \left(-i \omega_{0} T_{0}\right)$,

$x_{21}\left(T_{0}, T_{2}\right)=A_{21}\left(T_{2}\right) \exp \left(i \omega_{0} T_{0}\right)+\bar{A}_{21}\left(T_{2}\right) \exp \left(-i \omega_{0} T_{0}\right)$,

where it is convenient to express $A_{11}$ and $A_{21}$ in the polar form:

$$
\begin{aligned}
& A_{11}\left(T_{2}\right)=\frac{1}{2} a_{1}\left(T_{2}\right) \exp \left[i \theta_{1}\left(T_{2}\right)\right], \\
& A_{21}\left(T_{2}\right)=\frac{1}{2} a_{2}\left(T_{2}\right) \exp \left[i \theta_{2}\left(T_{2}\right)\right],
\end{aligned}
$$

where $a_{1}$ and $a_{2}$ are the amplitudes of Drive-I and Drive-II in the drive mode, respectively. $\theta_{1}$ and $\theta_{2}$ are the initial phases of Drive-I and Drive-II in the drive mode, respectively. Next, substituting equations (11) and (12) into (10), the averaging equations of amplitude and phase can be obtained from the solvability condition that does not produce a secular term.

$$
\begin{aligned}
\dot{a}_{1}= & -\frac{\widehat{\alpha}_{3} a_{1}}{2}+\frac{\widehat{\alpha}_{2} a_{2}}{2 \omega_{0}} \sin \phi+\frac{\widehat{\alpha}_{4} a_{2}}{2} \cos \phi-\frac{2 \widehat{\gamma}_{2} V_{d} V_{a}}{\omega_{0}} \sin \theta_{1}, \\
\dot{\theta}_{1}= & \frac{\widehat{\alpha}_{1}-\widehat{\omega}_{1}^{2}-\sigma_{1}-\left(4 \widehat{\gamma}_{1} V_{d}^{2} / g_{0}^{3}\right)}{2 \omega_{0}}-\frac{\widehat{\alpha}_{2} a_{2}}{2 \omega_{0} a_{1}} \cos \phi+\frac{\widehat{\alpha}_{4} a_{2}}{2 a_{1}} \sin \phi-\frac{3 \widehat{\gamma}_{1} V_{d}^{3} a_{1}^{2}}{g_{0}^{5} \omega_{0}}-\frac{2 \widehat{\gamma}_{2} V_{d} V_{a}}{\omega_{0} a_{1}} \cos \theta_{1}, \\
\dot{a}_{2}= & -\frac{\widehat{\beta}_{4} a_{2}}{2}-\frac{\widehat{\beta}_{1} a_{1}}{2 \omega_{0}} \sin \phi+\frac{\widehat{\beta}_{3} a_{1}}{2} \cos \phi, \\
\dot{\phi}= & -\frac{\widehat{\beta}_{1} a_{1}}{2 \omega_{0} a_{2}} \cos \phi+\frac{\widehat{\beta}_{2}-\widehat{\omega}_{2}^{2}+\sigma_{2}-\sigma_{1}}{2 \omega_{0}}-\frac{\widehat{\beta}_{3} a_{1}}{2 a_{2}} \sin \phi-\frac{\widehat{\alpha}_{1}-\widehat{\omega}_{1}^{2}-\sigma_{1}-\left(4 \widehat{\gamma}_{1} V_{d}^{2} / g_{0}^{3}\right)}{2 \omega_{0}} \\
& +\frac{\widehat{\alpha}_{2} a_{2}}{2 \omega_{0} a_{1}} \cos \phi-\frac{\widehat{\alpha}_{4} a_{2}}{2 a_{1}} \sin \phi+\frac{3 \widehat{\gamma}_{1} V_{d}^{3} a_{1}^{2}}{g_{0}^{5} \omega_{0}}+\frac{2 \widehat{\gamma}_{2} V_{d} V_{a}}{\omega_{0} a_{1}} \cos \theta_{1},
\end{aligned}
$$


where $\phi=\theta_{2}-\theta_{1}$.
In equation (13), let $\left(\dot{a}_{1}=0, \dot{\theta}_{1}=0, \dot{a}_{2}=0, \dot{\phi}=0\right)$. Then, two bifurcation equations of driving direction amplitude can be obtained by eliminating $\theta_{1}$ and $\phi$.

$$
\begin{aligned}
& a_{2}^{2}\left[4 \widehat{\beta}_{1} \widehat{\beta}_{2}-4 \widehat{\beta}_{1}\left(\sigma_{1}-\sigma_{2}\right)+4 \widehat{\beta}_{3} \widehat{\beta}_{4} \omega_{0}^{2}-4 \widehat{\beta}_{1} \widehat{\omega}_{2}^{2}\right]^{2}+a_{2}^{2} \omega_{0}^{2}\left[4 \widehat{\beta}_{2} \widehat{\beta}_{3}-4 \widehat{\beta}_{1} \widehat{\beta}_{4}-4 \widehat{\beta}_{3}\left(\sigma_{1}-\sigma_{2}\right)-4 \widehat{\beta}_{3} \widehat{\omega}_{2}^{2}\right]^{2}=16 a_{1}^{2}\left(\widehat{\beta}_{1}^{2}+\widehat{\beta}_{3}^{2} \omega_{0}^{2}\right)^{2}, \\
& g_{0}^{10} \omega_{0}^{2}\left[4 \widehat{\alpha}_{3} \widehat{\beta}_{1}^{2} a_{1}^{2}-4 \widehat{\alpha}_{4} \widehat{\beta}_{1} \widehat{\beta}_{2} a_{2}^{2}-4 \widehat{\alpha}_{2} \widehat{\beta}_{2} \widehat{\beta}_{3} a_{2}^{2}+4 \widehat{\alpha}_{2} \widehat{\beta}_{1} \widehat{\beta}_{4} a_{2}^{2}-4 \widehat{\alpha}_{4} \widehat{\beta}_{1} a_{2}^{2} \sigma_{2}-4 \widehat{\alpha}_{2} \widehat{\beta}_{3} a_{2}^{2} \sigma_{2}+4 \widehat{\alpha}_{4} \widehat{\beta}_{1} \omega_{0}^{2} a_{2}^{2}\right. \\
& \left.\quad+4 \widehat{\alpha}_{2} \widehat{\beta}_{3} \omega_{0}^{2} a_{2}^{2}+4 \widehat{\alpha}_{3} \widehat{\beta}_{3}^{2} \omega_{0}^{2} a_{1}^{2}-4 \widehat{\alpha}_{4} \widehat{\beta}_{3} \widehat{\beta}_{4} \widehat{\omega}_{0}^{2} a_{2}^{2}-4 \widehat{\alpha}_{4} \widehat{\beta}_{1} \widehat{\omega}_{1}^{2} a_{2}^{2}-4 \widehat{\alpha}_{2} \widehat{\beta}_{3} \widehat{\omega}_{1}^{2} a_{2}^{2}+4 \widehat{\alpha}_{4} \widehat{\beta}_{1} \widehat{\omega}_{2}^{2} a_{2}^{2}+4 \widehat{\alpha}_{2} \widehat{\beta}_{3} \widehat{\omega}_{2}^{2} a_{2}^{2}\right]^{2} \\
& \quad+\left[-4 g_{0}^{5} \widehat{\alpha}_{1} \widehat{\beta}_{1}^{2} a_{1}^{2}+4 g_{0}^{5} \widehat{\alpha}_{2} \widehat{\beta}_{1} \widehat{\beta}_{2} a_{2}^{2}+24 V_{d}^{2} \widehat{\beta}_{1}^{2} \widehat{\gamma}_{1} a_{1}^{4}+16 g_{0}^{2} V_{d}^{2} \widehat{\beta}_{1}^{2} \widehat{\gamma}_{1} a_{1}^{2}+4 g_{0}^{5} \widehat{\alpha}_{2} \widehat{\beta}_{1} \sigma_{2} a_{2}^{2}-4 g_{0}^{5} \widehat{\alpha}_{2} \widehat{\beta}_{1} \omega_{0}^{2} a_{2}^{2}+4 g_{0}^{5} \widehat{\beta}_{1}^{2} \omega_{0}^{2} a_{1}^{2}\right. \\
& \quad-4 g_{0}^{5} \widehat{\alpha}_{4} \widehat{\beta}_{2} \widehat{\beta}_{3} \omega_{0}^{2} a_{2}^{2}-4 g_{0}^{5} \widehat{\alpha}_{1} \widehat{\beta}_{3}^{2} \omega_{0}^{2} a_{1}^{2}+4 g_{0}^{5} \widehat{\alpha}_{4} \widehat{\beta}_{1} \widehat{\beta}_{4} \omega_{0}^{2} a_{2}^{2}+4 g_{0}^{5} \widehat{\alpha}_{2} \widehat{\beta}_{3} \widehat{\beta}_{4} \omega_{0}^{2} a_{2}^{2}+24 V_{d}^{2} \widehat{\beta}_{3}^{2} \widehat{\gamma}_{1} \omega_{0}^{2} a_{1}^{4}+16 g_{0}^{2} V_{d}^{2} \widehat{\beta}_{3}^{2} \widehat{\gamma}_{1} \omega_{0}^{2} a_{1}^{2} \\
& \quad-4 g_{0}^{5} \widehat{\alpha}_{4} \widehat{\beta}_{3} \sigma_{2} \omega_{0}^{2} a_{2}^{2}+4 g_{0}^{5} \widehat{\alpha}_{4} \widehat{\beta}_{3} \omega_{0}^{4} a_{2}^{2}+4 g_{0}^{5} \widehat{\beta}_{3}^{2} \omega_{0}^{4} a_{1}^{2}+4 g_{0}^{5} \widehat{\alpha}_{2} \widehat{\beta}_{1} \omega_{1}^{2} a_{2}^{2} \\
& \left.\quad-4 g_{0}^{5} \widehat{\alpha}_{4} \widehat{\beta}_{3} \omega_{0}^{2} \omega_{1}^{2} a_{2}^{2}-4 g_{0}^{5} \widehat{\alpha}_{2} \widehat{\beta}_{1} \omega_{2}^{2} a_{2}^{2}+4 g_{0}^{5} \widehat{\alpha}_{4} \widehat{\beta}_{3} \omega_{0}^{2} \omega_{2}^{2} a_{2}^{2}\right]^{2}=256 g_{0}^{10} V_{a}^{2} V_{d}^{2}\left(\widehat{\beta}_{1}^{2}+\widehat{\beta}_{3} \omega_{0}^{2}\right)^{2} \widehat{\gamma}_{2}^{2} a_{1}^{2} .
\end{aligned}
$$

The nonlinear coupling equations (14) and (15) are solved iteratively by Newton-Raphson method and pseudoarc-length continuation. Finally, the response of the amplitudes $a_{1}$ and $a_{2}$ can be obtained.

In order to obtain the sense response under the electrostatic force nonlinearity in the drive direction, the complex exponential method is used to solve the dynamic equations of sense direction. The approximate solution of
Drive-II $\left(x_{2}\right)$ is $a_{2} \cos \left(\omega_{0} t-\theta_{2}\right)$, and the Coriolis force can be expressed as $2 m_{2} \Omega_{z} a_{2} \omega_{0} \sin \left(\omega_{0} t-\theta_{2}\right)$. Let $f_{c}$ be the amplitude of Coriolis force; then $f_{c}=2 m_{2} \Omega_{z} a_{2} \omega_{0}$. Obviously, $f_{c}$ is related to the excitation frequency and the amplitude of $a_{2}$ in the driving direction. In equation (16), $b_{1}$ and $b_{2}$ are the steady-state response amplitudes of the sense direction, respectively.

$$
\left\{\begin{array}{l}
b_{1}=\frac{f_{c}\left[\left(c_{5}+c_{6}\right)^{2} \omega_{0}^{2}+\left(k_{5}+k_{6}-m_{3} \omega_{0}^{2}\right)^{2}\right]^{(1 / 2)}}{\left\{\begin{array}{l}
{\left[-2 c_{5} k_{5} \omega_{0}+\left(c_{5}+c_{6}\right)\left(k_{4}+k_{5}-m_{2} \omega_{0}^{2}\right) \omega_{0}+\left(c_{4}+c_{5}\right)\left(k_{5}+k_{6}-m_{3} \omega_{0}^{2}\right) \omega_{0}\right]^{2}} \\
+\left[-k_{5}^{2}+c_{5}^{2} \omega_{0}^{2}-\left(c_{4}+c_{5}\right)\left(c_{5}+c_{6}\right) \omega_{0}^{2}+\left(k_{4}+k_{5}-m_{2} \omega_{0}^{2}\right)\left(k_{5}+k_{6}-m_{3} \omega_{0}^{2}\right)\right]^{2}
\end{array}\right\}^{(1 / 2)}} \\
b_{2}=\frac{f_{c}\left(k_{5}^{2}+c_{5}^{2} \omega_{0}^{2}\right)^{(1 / 2)}}{\left\{\begin{array}{l}
{\left[-2 c_{5} k_{5} \omega_{0}+\left(c_{5}+c_{6}\right)\left(k_{4}+k_{5}-m_{2} \omega_{0}^{2}\right) \omega_{0}+\left(c_{4}+c_{5}\right)\left(k_{5}+k_{6}-m_{3} \omega_{0}^{2}\right) \omega_{0}\right]^{2}} \\
+\left[-k_{5}^{2}+c_{5}^{2} \omega_{0}^{2}-\left(c_{4}+c_{5}\right)\left(c_{5}+c_{6}\right) \omega_{0}^{2}+\left(k_{4}+k_{5}-m_{2} \omega_{0}^{2}\right)\left(k_{5}+k_{6}-m_{3} \omega_{0}^{2}\right)\right]^{2}
\end{array}\right.}
\end{array}\right.
$$

\section{Influence of Electrostatic Nonlinearity on Dynamic Behaviours of MVG}

In this section, the influence of driving comb spacing $g_{0}$ and DC bias voltage $V_{d}$ on the dynamic behaviour of the MVG is analysed. The values of physical parameters of MVG are shown in Table 1 [18].

4.1. Effect of Comb Spacing on Dynamic Response. The influence of comb spacing on the dynamic performance is considered. The calculation parameters are set to $V_{d}=40 \mathrm{~V}$,
$V_{a}=20 \mathrm{~V}$, and $\varepsilon=1$, which are DC bias voltage, AC voltage, and small parameter, respectively. According to the parameters in Table 1 , the first- and second-order natural frequencies in the driving direction are $\omega_{1}=30940.11(\mathrm{rad} / \mathrm{s}) \quad$ and $\quad \omega_{2}=31880.86(\mathrm{rad} / \mathrm{s})$, respectively.

Figures 4 and 5 show the amplitude frequency responses of Drive-I $\left(x_{1}\right)$ and Drive-II $\left(x_{2}\right)$ under different comb spacing values. In order to verify the analytical solution obtained by the multiple time scales method, the RungeKutta method is used to solve equation (5). A series of numerical results are obtained. Compared with the 
TABle 1: Physical parameters of the 4-DOF MVG.

\begin{tabular}{lccccc}
\hline Mass & Value $(\mathrm{kg})$ & Damping & Value $(\mathrm{Ns} / \mathrm{m})$ & Stiffness coefficient & Value $(\mathrm{N} / \mathrm{m})$ \\
\hline$m_{1}$ & $2.36 \times 10^{-7}$ & $c_{1}$ & $10^{-4}$ & $k_{1}$ & 224.7 \\
$m_{2}$ & $2.74 \times 10^{-7}$ & $c_{2}$ & $5 \times 10^{-6}$ & $k_{2}$ & 8.2 \\
$m_{f}$ & $5.23 \times 10^{-8}$ & $c_{3}$ & $2 \times 10^{-4}$ & $k_{3}$ & 313.8 \\
$m_{3}$ & $1.35 \times 10^{-7}$ & $c_{4}$ & $10^{-4}$ & $k_{4}$ & 260.9 \\
- & - & $c_{5}$ & $5 \times 10^{-6}$ & $k_{5}$ & 9.5 \\
- & - & $c_{6}$ & $2 \times 10^{-4}$ & $k_{6}$ & 123.7 \\
\hline
\end{tabular}

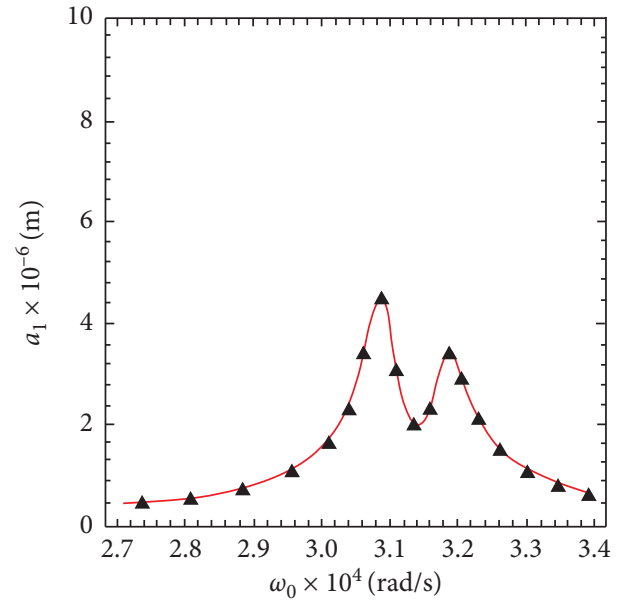

- Analytical solution

\ Numerical solution

(a)

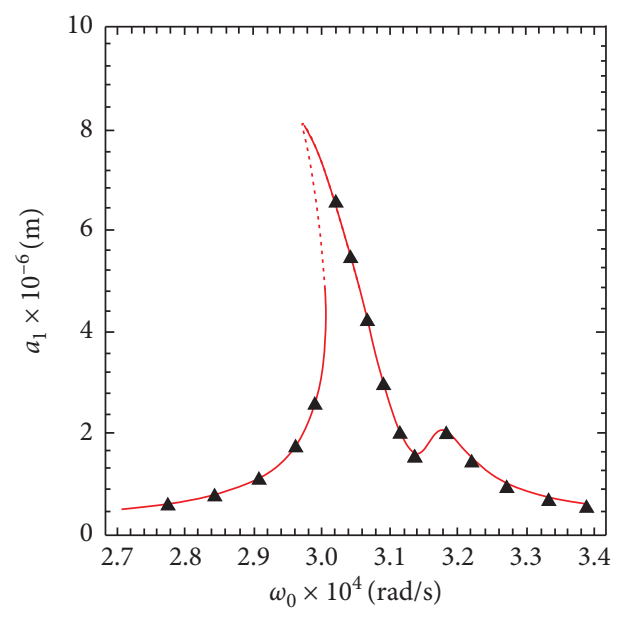

- Analytical solution

\ Numerical solution

(c)

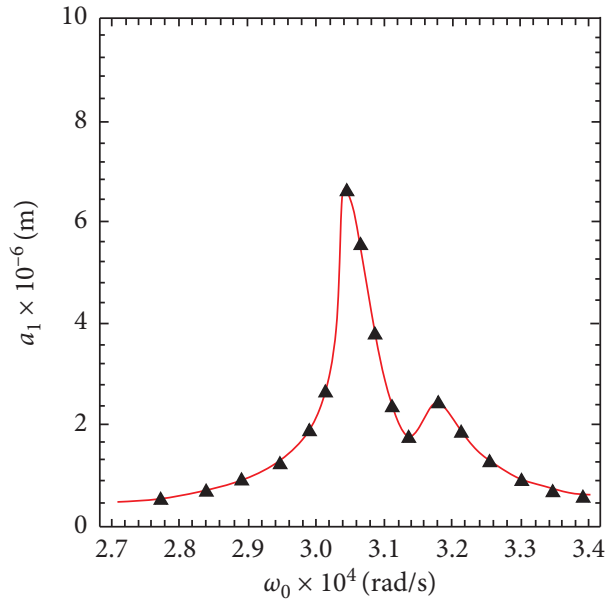

- Analytical solution

- Numerical solution

(b)

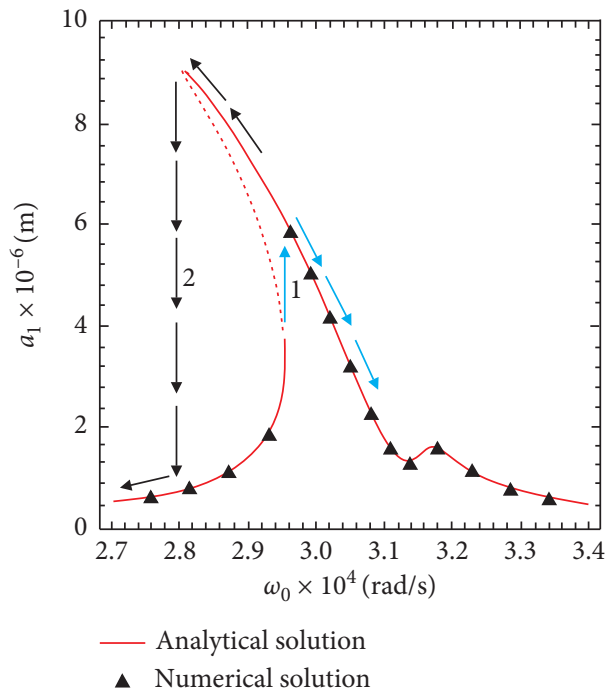

(d)

Figure 4: Amplitude frequency response of Drive-I. (a) $g_{0}=15.0 \mu \mathrm{m}$. (b) $g_{0}=9.0 \mu \mathrm{m}$. (c) $g_{0}=8.0 \mu \mathrm{m}$. (d) $g_{0}=7.0 \mu \mathrm{m}$.

analytical solution, it can be seen that there is a good agreement. The other analytical results are also verified by numerical simulation in the following parts.

As is shown in Figure 4, when comb spacing $g_{0}$ is reduced from $15.0 \mu \mathrm{m}$ to $9 \mu \mathrm{m}$, the amplitude of the first resonance peak of Drive-I increases greatly and slightly bends to the left. It is shown that nonlinear electrostatic force has weak softening characteristics. Also, the sensitivity at the original first resonance frequency is decreased by $22.7 \%$, and the sensitivity at the original second resonance frequency is also greatly reduced.

When $g_{0}$ decreases to $8 \mu \mathrm{m}$, the amplitude of the first resonance peak increases further and bends to the left obviously. The typical nonlinear characteristics such as 


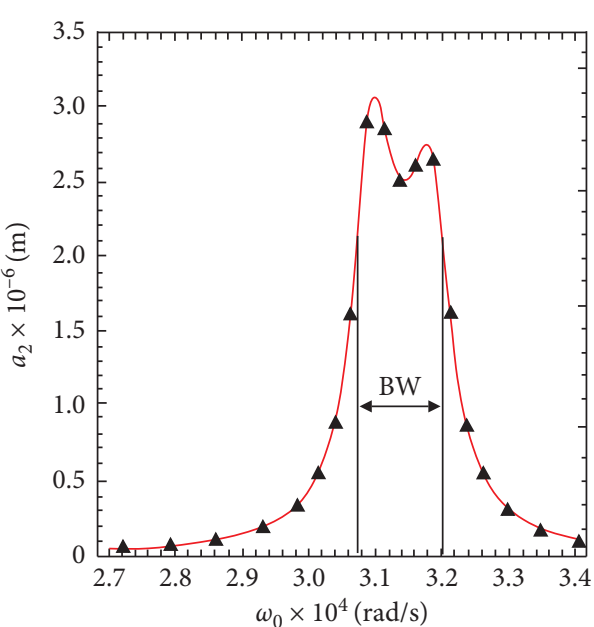

— Analytical solution

- Numerical solution

(a)

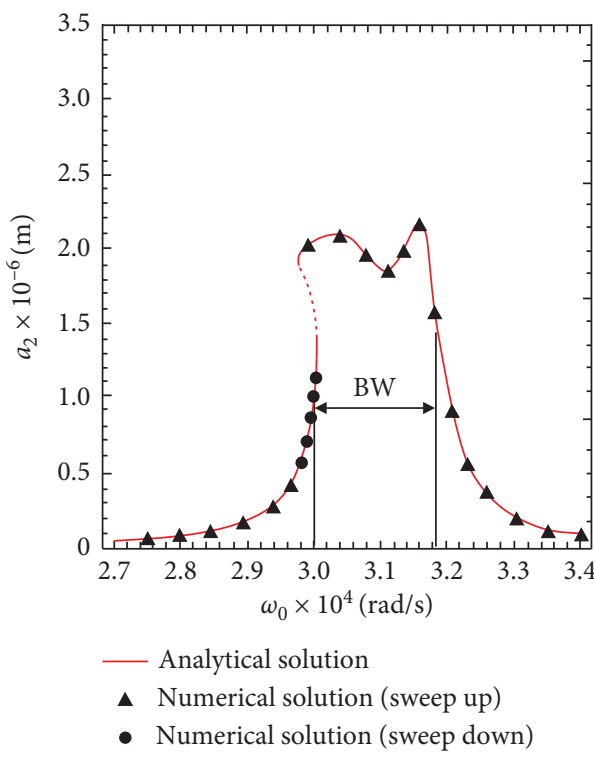

(c)

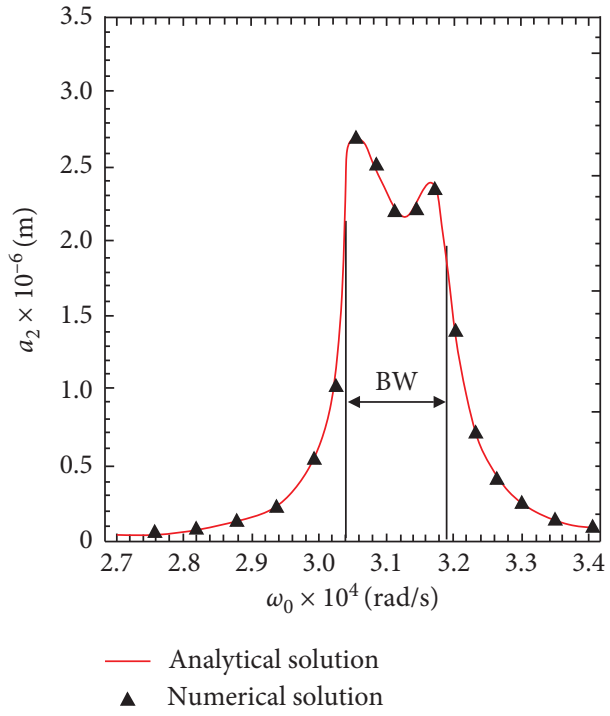

(b)

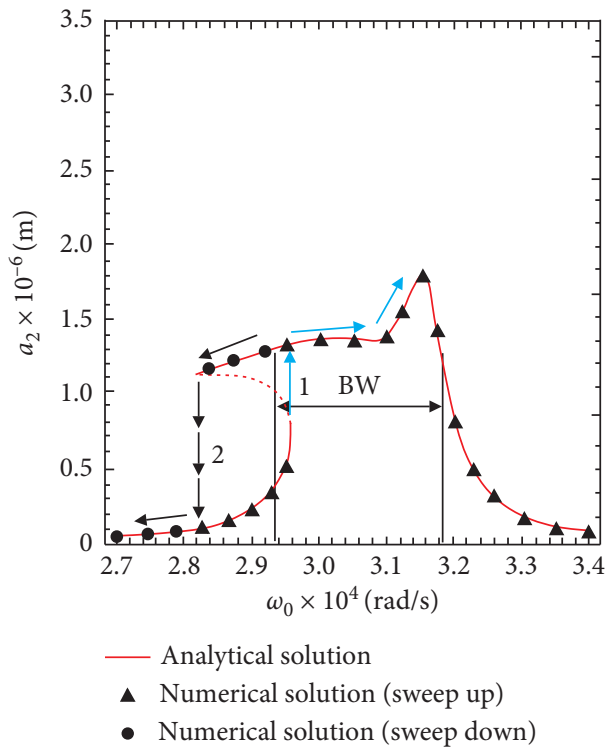

(d)

Figure 5: Amplitude frequency response of Drive-II. (a) $g_{0}=15.0 \mu \mathrm{m}$. (b) $g_{0}=9.0 \mu \mathrm{m}$. (c) $g_{0}=8.0 \mu \mathrm{m}$. (d) $g_{0}=7.0 \mu \mathrm{m}$.

multistable solution, amplitude jump, and frequency offset appear. Compared with $g_{0}=15.0 \mu \mathrm{m}$, the sensitivity of the first resonance frequency decreased by $39.5 \%$, the second resonance frequency shifted slightly to the left, and the sensitivity of the original second resonance frequency decreased by $44.2 \%$.

If $g_{0}$ subsequently decreases to $7 \mu \mathrm{m}$, the nonlinear softening property is further enhanced and the instability region is further expanded. As seen in Figure 4(d), there is obviously jump phenomenon. Arrows 1 (blue) indicate that the route of amplitude changes when the frequency $\omega_{0}$ increases. By contrast, when $\omega_{0}$ gradually decreases from high frequency to low frequency, the change route of amplitude is indicated by arrows 2 (black). It is also the one of the specific characteristics in the nonlinear dynamic systems. Owing to the amplitude jump, multistable solution, and frequency offset, the stability of sensitivity near the original first resonance frequency is destroyed. There is another steady-state solution far below the peak value of the first natural frequency; it is due to the dependence of the nonlinear system on the initial conditions. When the working frequency increases from low frequency to resonance frequency, the system is periodic motion with small amplitude; when the working frequency is reduced from high frequency to resonance frequency, the system is periodic motion with large amplitude.

It should be noted that the maximum amplitude of Drive-I has exceeded the spacing value when the comb spacing is less than $8 \mu \mathrm{m}$. It may lead to the phenomenon of pull-in and even cause the damage of driving comb. That is, the large amplitude jump of the response not only affects the stability of the dynamic performance of the 


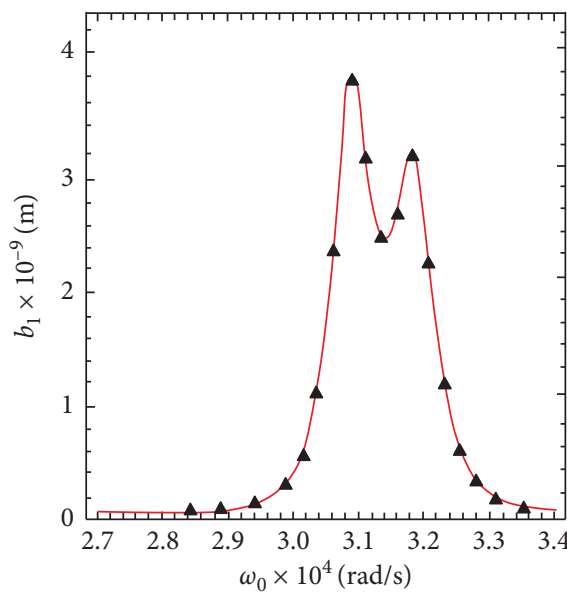

- Analytical solution

- Numerical solution

(a)

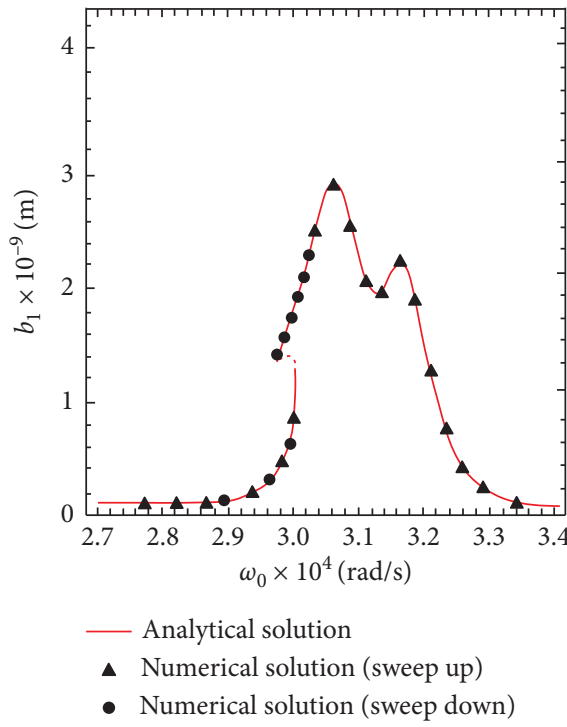

(c)

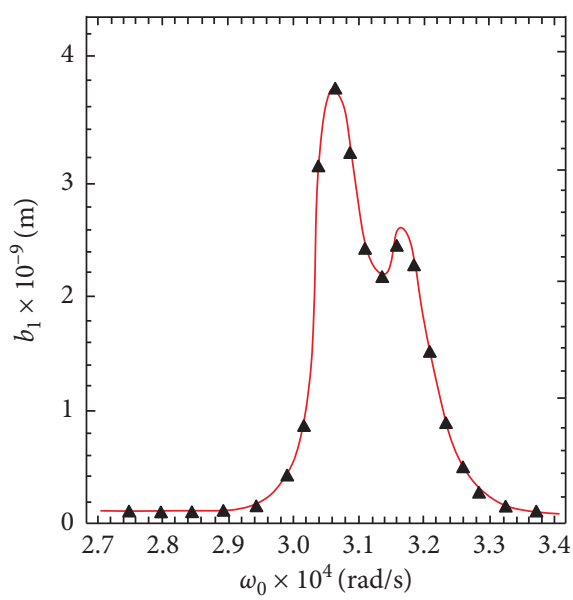

- Analytical solution

- Numerical solution

(b)

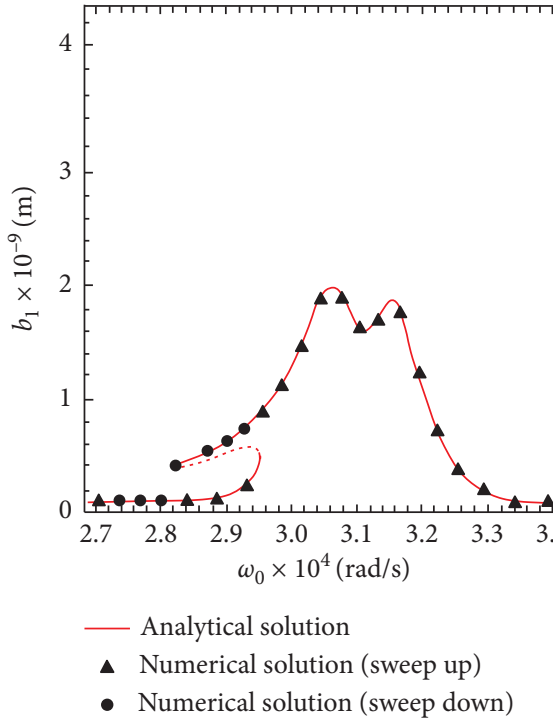

(d)

Figure 6: Amplitude frequency response of Sense-I. (a) $g_{0}=15.0 \mu \mathrm{m}$. (b) $g_{0}=9.0 \mu \mathrm{m}$. (c) $g_{0}=8.0 \mu \mathrm{m}$. (d) $g_{0}=7.0 \mu \mathrm{m}$.

MVG but also leads to the damage of the microbeam in some cases.

As shown in Figure 5, the response of Drive-II is different from that of Drive-I. When $g_{0}$ is equal to $9.0 \mu \mathrm{m}$, the amplitude frequency response shows the weak softening characteristics. The response curve of the first and second resonances slightly bends to the left, but the maximum amplitude is reduced significantly. When $g_{0}$ is reduced to $8 \mu \mathrm{m}$, the response curve shows the nonlinear softening characteristics. The nonlinear characteristics such as multistable solution, amplitude jump, and frequency offset appear near the first peak. The electrostatic nonlinearity becomes very sensitive to the change of comb spacing, as illustrated in Figures 5(c) and 5(d).

The $\mathrm{BW}$ region in Figure 5 represents the $3 \mathrm{~dB}$ bandwidth of the Drive-II response. As the comb spacing decreases, the output response bandwidth gradually increases. But the frequency corresponding to the bandwidth slightly shifts to the left due to the nonlinear frequency offset. In the bandwidth range, the sensitivity of the response decreases greatly with the enhancement of the nonlinearity. The multistable solution caused by the softening effect of the electrostatic nonlinearity also appears in this range. It means that sensitivity is unstable in the bandwidth range. If the excitation frequency of MVG is changed from high to low, its amplitude will jump greatly. This jump of large amplitude may damage the microbeam and then lead the MVG to not be able to operate normally.

Combining equations (14)-(16), the relationship between the steady-state amplitude of the sense mode and the driving frequency can be obtained. The amplitude frequency responses of the first and second primary resonances are shown in Figures 6 and 7. 


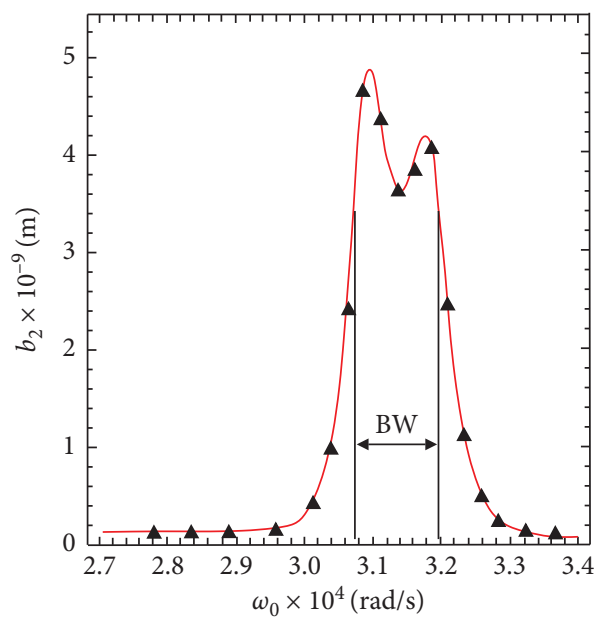

- Analytical solution

\ Numerical solution

(a)

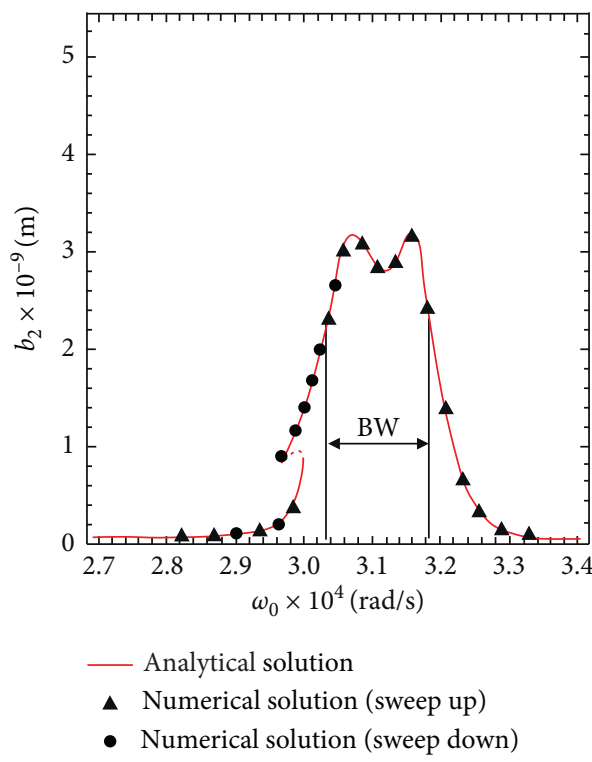

(c)

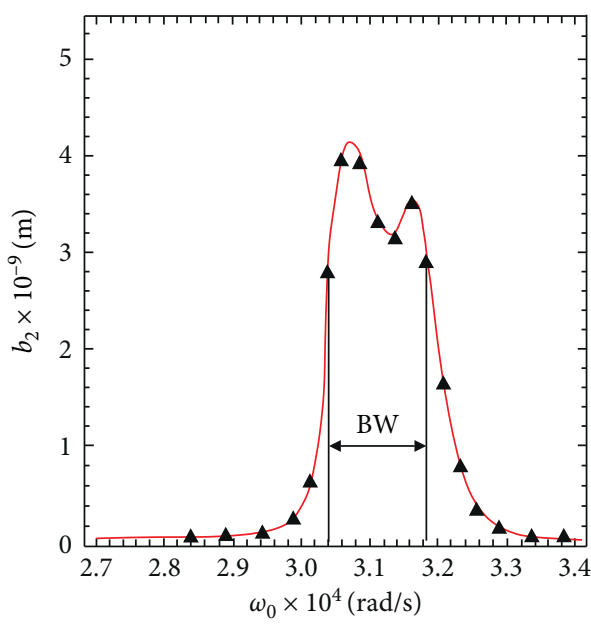

- Analytical solution

- Numerical solution

(b)

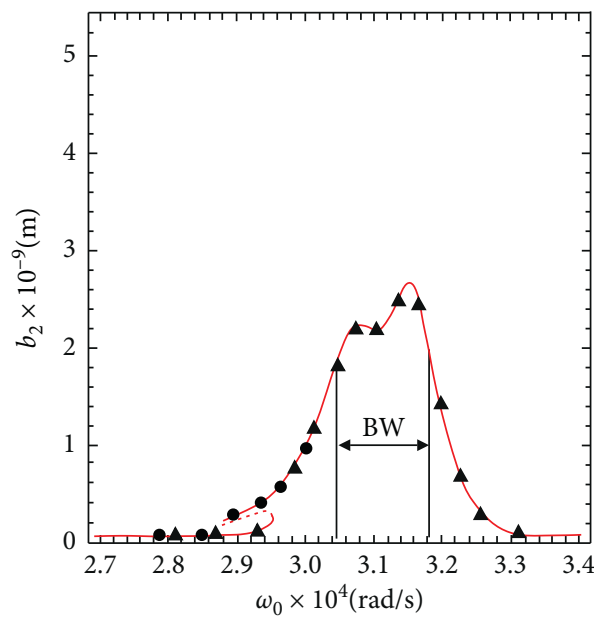

- Analytical solution

- Numerical solution (sweep up)

- Numerical solution (sweep down)

(d)

Figure 7: Amplitude frequency response of Sense-II. (a) $g_{0}=15.0 \mu \mathrm{m}$. (b) $g_{0}=9.0 \mu \mathrm{m}$. (c) $g_{0}=8.0 \mu \mathrm{m}$. (d) $g_{0}=7.0 \mu \mathrm{m}$.

It can be seen from Figures 6 and 7 that the amplitude frequency responses of Sense-I and Sense-II are similar to that of Drive-II. When $g_{0}=9.0 \mu \mathrm{m}$, as shown in Figure 7(b), the amplitude frequency response shows the nonlinear softening characteristics. Then, the slight change of $g_{0}$ makes the shape of the response curve change obviously.

Furthermore, the amplitude frequency response has typical nonlinear behaviours such as multistable solution, amplitude jump, and frequency offset in Figures 6(c), 6(d), $7(\mathrm{c})$, and $7(\mathrm{~d})$. It can be seen that the electrostatic force nonlinearity has strong influence on its amplitude frequency response.

The area marked by BW is the sense bandwidth of the MVG in Figure 7. The bandwidth of output response is basically unchanged. But the sensitivity is greatly reduced with the enhancement of the nonlinear electrostatic force. The region of multistable solution is outside the sense bandwidth and does not affect the stability of sensitivity. Thus, when the MVG works in the sense bandwidth range, the instability behaviours such as amplitude jump will not appear even if there is a strong electrostatic force. It is due to the high robustness of the sense mode that the amplitude in the bandwidth range changes a little. The electrostatic force nonlinearity has little effect on the bandwidth of output response in the sense direction.

In order to reveal the influence of comb spacing on the dynamic characteristics of MVG more intuitively, the relation curve between comb spacing and Drive-I response amplitude is shown in Figure 8. Drive-II, Sense-I, and SenseII are similar to Drive-I, so they are neglected. When the 


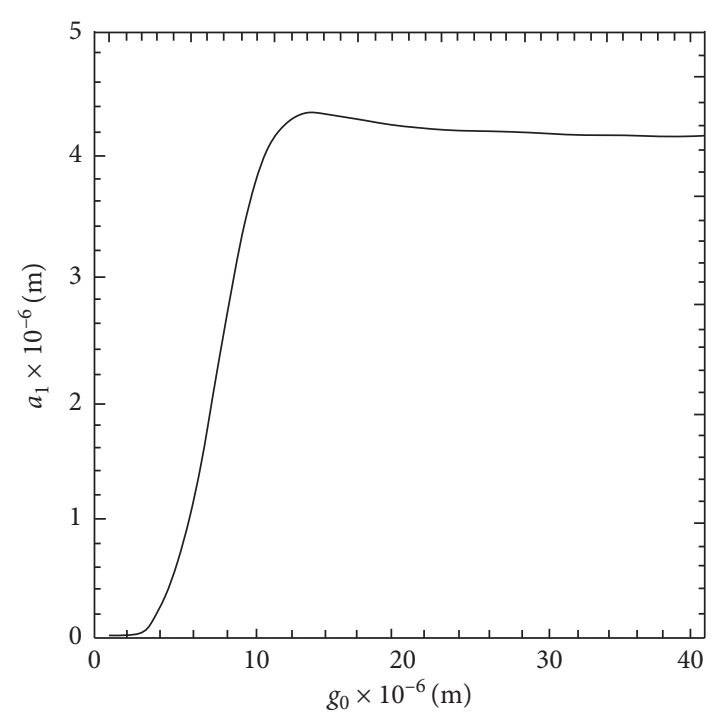

Figure 8: The influence of comb spacing on the amplitude of Drive-I.
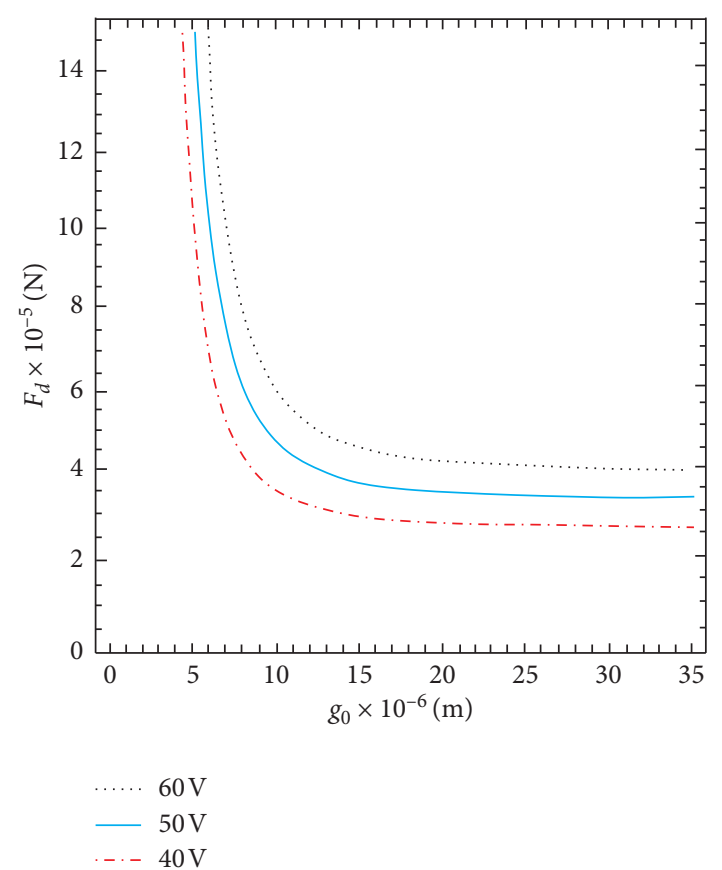

FIGURE 9: Relationship between comb spacing and electrostatic force.

excitation frequency is equal to the natural frequency of Drive-I, the amplitude of Drive-I increases rapidly with the increase of $g_{0}$. Then it decreases slightly after reaching the peak value and gradually reaches a stable value. That is, the amplitude of the system is no longer affected by the comb spacing.

There is a critical value for the comb spacing under a given voltage according to Figures 4-8. Dynamic behaviours of MVG are affected by electrostatic force nonlinearity when $g_{0}$ is less than the critical value. In this case, the softening characteristics are shown in the MVG system, and the response curve of the first resonance bends to the left, which leads to a significant reduction in the response amplitude at the original design natural frequency. The influence of softening characteristics produced by electrostatic force nonlinearity increases with the decrease of $g_{0}$. It results in a significant reduction in the sensitivity of the system, but the bandwidth is basically unaffected. When $g_{0}$ is larger than the critical value, the system is no longer affected by the comb spacing. The effectiveness of linear design is guaranteed in this case.

Figure 9 shows the relationship between comb spacing and electrostatic force under three different values of DC bias voltage. It can be seen that when $V_{d}=40 \mathrm{~V}$ and $g_{0}>15 \mu \mathrm{m}$, the variation of electrostatic force $F_{d}$ with increasing of $g_{0}$ is not obvious and tends to be a constant. This is corresponding to the fact that the amplitude of Drive-I tends to be constant when $g_{0}>15 \mu \mathrm{m}$ in Figure 8. It is also further verified that the dynamic characteristics of the MVG will be no longer affected by the comb spacing. When $g_{0}$ decreases from $15 \mu \mathrm{m}$ to $7 \mu \mathrm{m}$, the electrostatic force increases rapidly and the increase rate reaches $91.3 \%$. There is an obvious fringe effect between comb teeth in this case, which has a great impact on the electrostatic force [21]. Again, it can be confirmed that the softening characteristics of electrostatic force increase with the decrease of $g_{0}$ in Figure 4 .

As shown in Figure 10, the electric field distributions of the comb structure under different comb spacing are calculated by using the finite element software. When $g_{0}$ is in the range of $15 \mu \mathrm{m} \sim 20 \mu \mathrm{m}$, there is almost no change in the electric field at the edge of the comb. In other words, the fringe effect is not obvious, as shown in Figures 10(a) and 10(b). When $g_{0}$ decreases from $13 \mu \mathrm{m}$ to $12 \mu \mathrm{m}$, the electric field on the edge of the comb changes obviously. The equipotential surface of the electric field intersects with the fixed comb. The fringe effect is enhanced, which leads to the softening characteristics of the electrostatic force. When $g_{0}=5 \mu \mathrm{m}$, the electric field on the edge of the comb deforms greatly. It means that the strong fringe effect causes the MVG to show the strong softening characteristics of electrostatic force, which will lead to serious instability of the system. The simulation results are consistent with the previous theoretical analysis (as shown in Figures 4, 8, and 9), verifying the reliability of the theoretical analysis results.

Therefore, in order to avoid the pull-in phenomenon and ensure the high sensitivity and stability of Drive-I, the comb spacing should be greater than the critical value when designing comb spacing of MVG. It should be considered that the value cannot be too large because of the compactness of the structure.

According to the above analysis, the critical value of driving comb spacing in the MVG can be determined by nonlinear dynamic analysis. Based on this, the comb spacing could be decreased as small as possible under the premise of effectiveness of linear design. This study provides a reliable basis of determining comb spacing of MVG.

4.2. Effect of DC Bias Voltage on Dynamics Response. It can be seen from the previous section that the response 


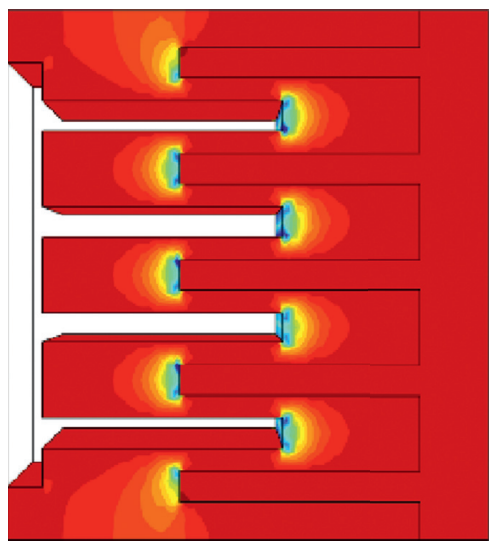

(a)

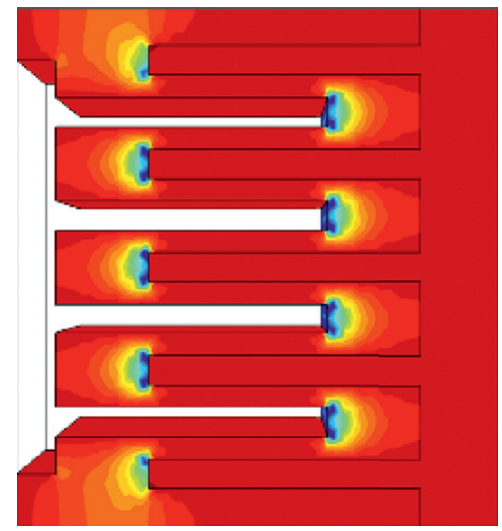

(d)

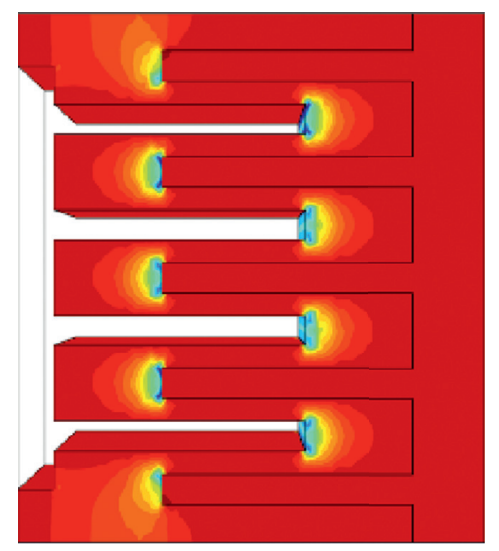

(b)

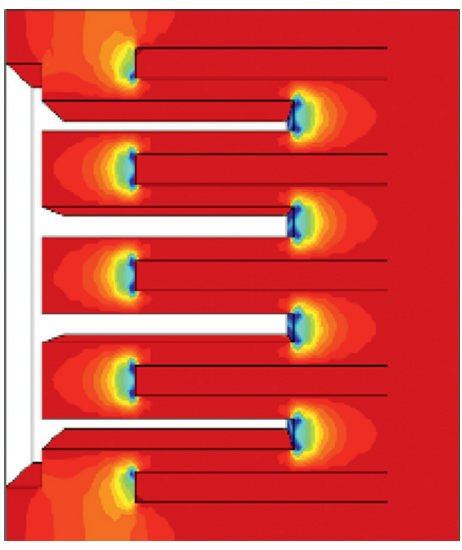

(c)

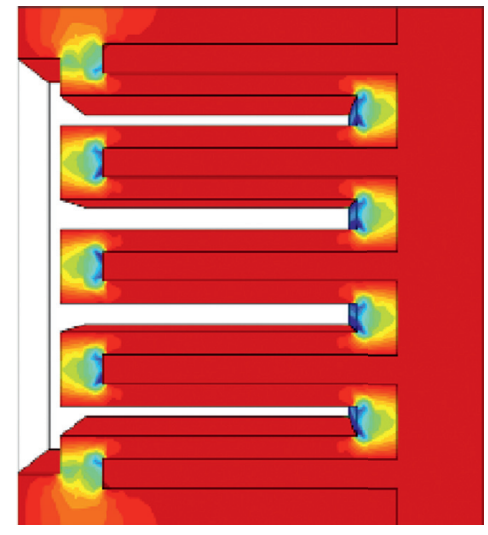

(e)

Figure 10: Electric field distribution of comb structure when $V_{d}=40 \mathrm{~V}$. (a) $g_{0}=20.0 \mu \mathrm{m}$. (b) $g_{0}=15.0 \mu \mathrm{m}$. (c) $g_{0}=13.0 \mu \mathrm{m}$. (d) $g_{0}=12.0 \mu \mathrm{m}$. (e) $g_{0}=5.0 \mu \mathrm{m}$.

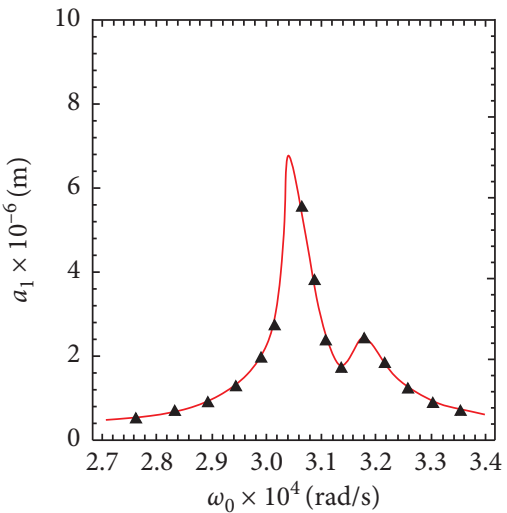

- Analytical solution

\ Numerical solution

(a)

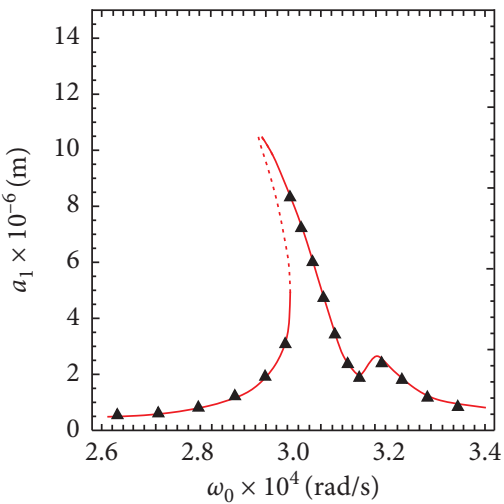

- Analytical solution

- Numerical solution

(b)

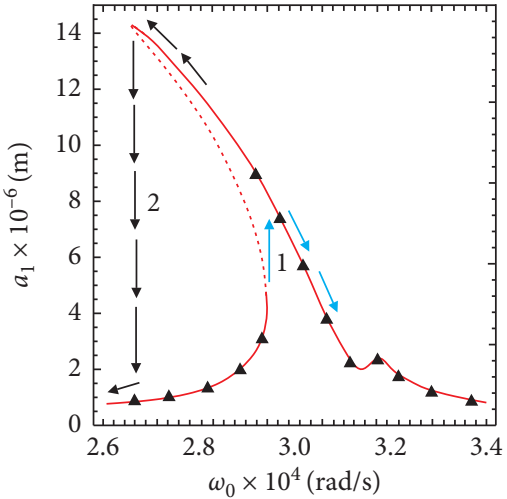

- Analytical solution

- Numerical solution

(c)

Figure 11: Amplitude frequency response of Drive-I. (a) $V_{d}=40 \mathrm{~V}$. (b) $V_{d}=50 \mathrm{~V}$. (c) $V_{d}=60 \mathrm{~V}$.

curves of the system begin to show softening characteristics when $V_{d}=40 \mathrm{~V}$ and $g_{0}=9.0 \mu \mathrm{m}$. Then the influence of DC bias voltage on the dynamics response of 4DOF MVG is studied when the comb spacing $g_{0}$ is $9.0 \mu \mathrm{m}$. Figures 11-14 show the amplitude frequency response of drive mode and sense mode under different DC bias voltage, respectively.

When the comb spacing $g_{0}=9.0 \mu \mathrm{m}$ and the DC bias voltage $V_{d}$ increases from $40 \mathrm{~V}$ to $60 \mathrm{~V}$, the maximum amplitude of Drive-I increases greatly. The response curves 


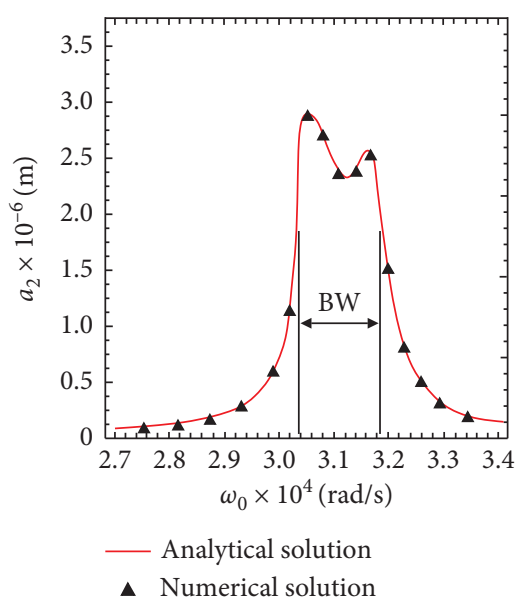

(a)

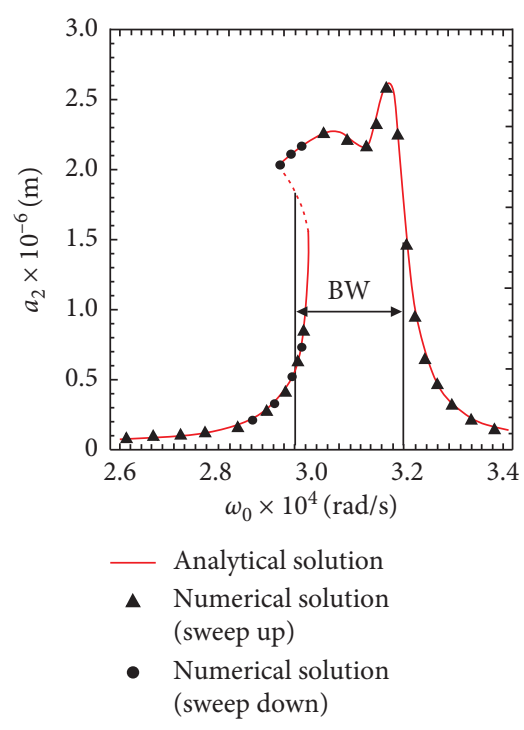

(b)

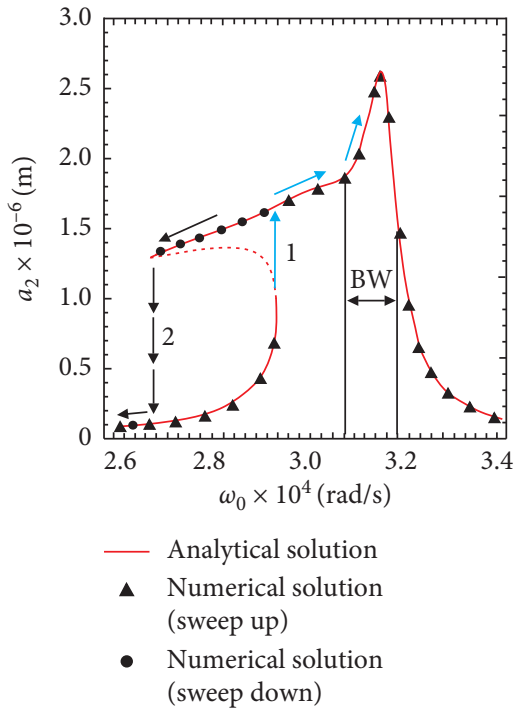

(c)

Figure 12: Amplitude frequency response of Drive-II. (a) $V_{d}=40 \mathrm{~V}$. (b) $V_{d}=50 \mathrm{~V}$. (c) $V_{d}=60 \mathrm{~V}$.

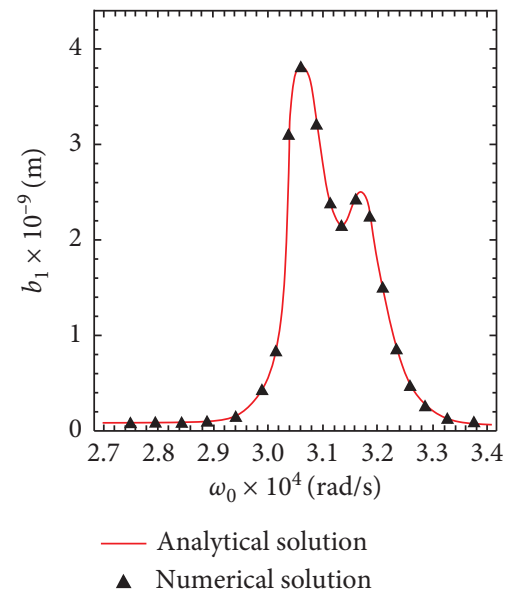

(a)

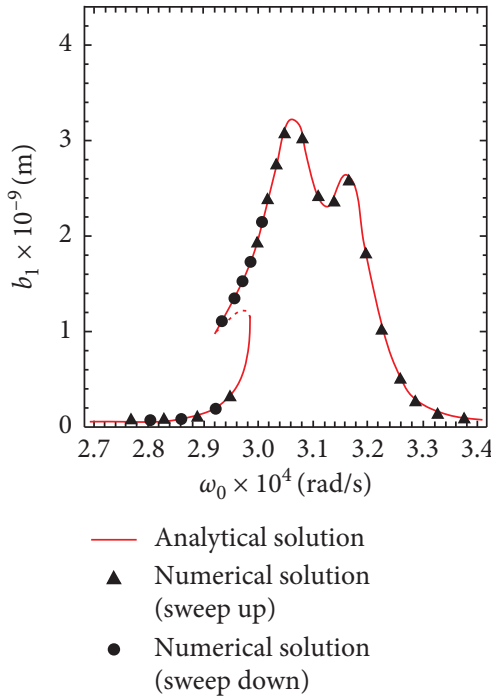

(b)

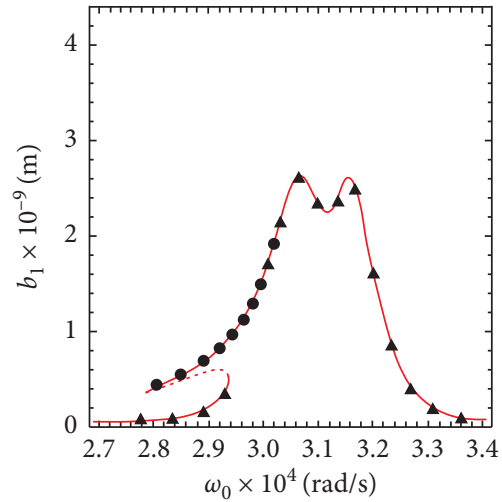

- Analytical solution

- Numerical solution (sweep up)

- Numerical solution (sweep down)

(c)

Figure 13: Amplitude frequency response of Sense-I. (a) $V_{d}=40 \mathrm{~V}$. (b) $V_{d}=50 \mathrm{~V}$. (c) $V_{d}=60 \mathrm{~V}$.

of the first resonance frequency bend to the left gradually. Nonlinear characteristics such as multistable solution, amplitude jump, and frequency offset appear near the first peak. Compared with the original linear design, the sensitivity at the original first and second resonance frequencies is reduced, as shown in Figure 11.

As is shown in Figures 12-14, the influences of DC bias voltage on Drive-II, Sense-I, and Sense-II are similar. The influence of electrostatic force nonlinearity is strengthened with the increase of DC bias voltage. The bandwidth range corresponding to the unstable region is increased. However, the unstable region is more and more away the working bandwidth of the MVG. The amplitude jump is also significantly reduced, which does not have enough influence on the stability of the dynamic performance. Because the output response of Sense-II is the sensitivity of the MVG, an important conclusion is that the sensitivity of the MVG decreases greatly with the increase of DC bias voltage.

In order to analyse deeply the influence of DC bias voltage and comb spacing on the MVG, the influence of DC bias voltage on amplitude of Drive-I under different comb spacing is obtained when the excitation frequency is equal to the natural frequency of Drive-I, as shown in Figure 15.

In Figure 15, when $g_{0}$ is fixed, the amplitude of Drive-I increases until reaching the peak value with the increase of DC bias voltage and then decreases gradually. At the left of 


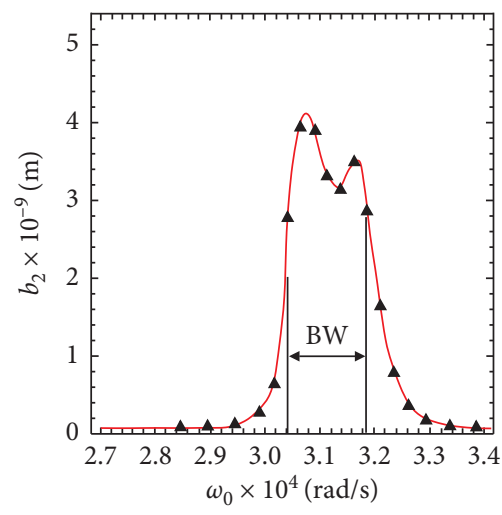

- Analytical solution

- Numerical solution

(a)

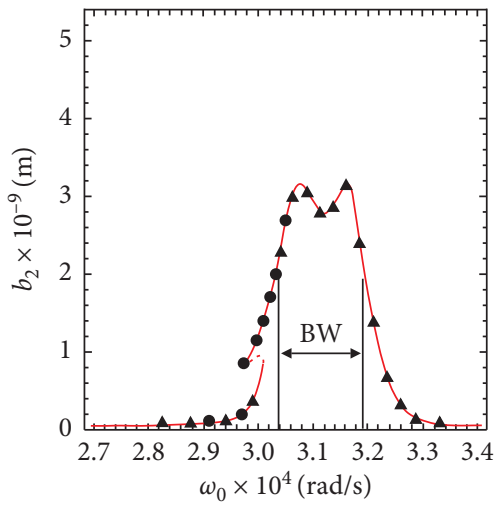

- Analytical solution

- Numerical solution (sweep up)

- Numerical solution (sweep down)

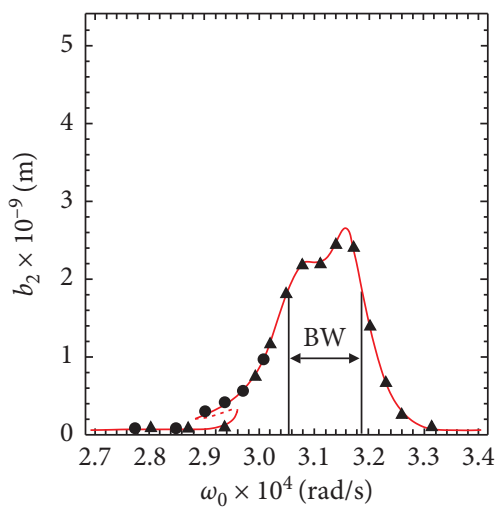

- Analytical solution

- Numerical solution (sweep up)

- Numerical solution (sweep down)

(b)

(c)

Figure 14: Amplitude frequency response of Sense-II. (a) $V_{d}=40 \mathrm{~V}$. (b) $V_{d}=50 \mathrm{~V}$. (c) $V_{d}=60 \mathrm{~V}$.

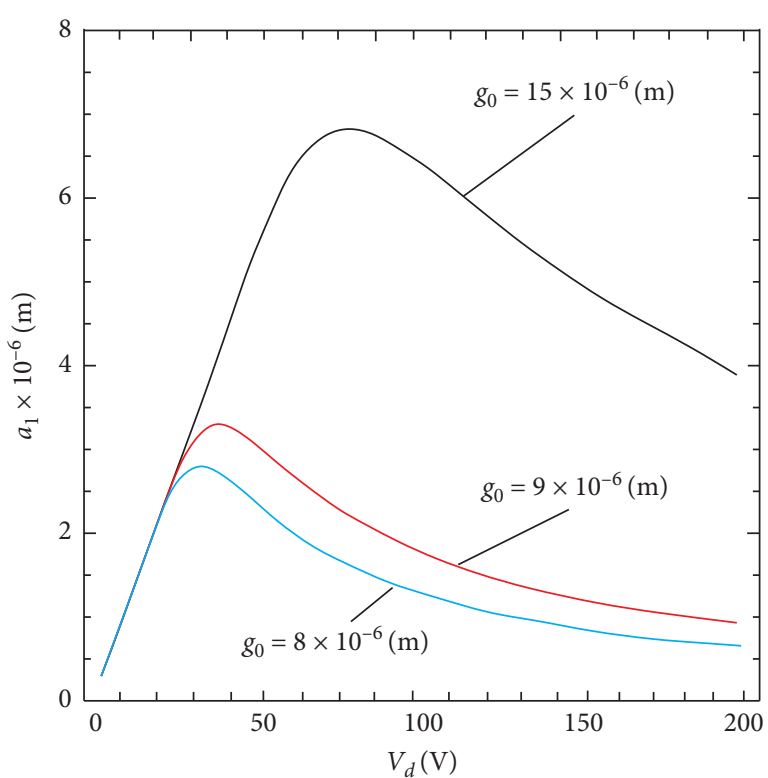

FIGURE 15: The influence of DC bias voltage on the amplitude of Drive-II under different comb spacing.

peak point, the electrostatic force is almost linear. The amplitude of response of Drive-I increases with the increase of bias DC voltage. At the right of the peak point, the nonlinear electrostatic force increases with the increase of the DC bias voltage. The softening characteristics caused by the electrostatic force nonlinearity result in the decrease of the natural frequency. The resonance point is shifted to the left. The amplitude of the response at the natural frequency decreases greatly. Obviously, the voltage corresponding to the peak point is the critical value where the electrostatic force nonlinearity produces effects. The voltage of the critical value increases with the increase of comb spacing. This phenomenon is also reflected in Figure 9.
Designing a higher DC bias voltage of driving comb is an important way to improve the sensitivity of MVG. However, when the voltage exceeds a critical value, the sensitivity of the MVG system is not increased but decreased because of the softening characteristics of electrostatic force. Therefore, it is necessary to consider the influence of DC bias voltage on electrostatic force nonlinearity in the parameters design of MVG. The above study provides a feasible idea for the determination of critical value of DC bias voltage in the MVG.

\section{Conclusions}

In this paper, a 4-DOF MVG with double drive mode and double sense mode was investigated by using multiple time scales method of nonlinear dynamics. The calculation results are also verified by finite element method and numerical simulation. The influence of DC bias voltage and comb spacing on the nonlinearity of electrostatic force was studied.

The DC bias voltage and the comb spacing have significant influence on the nonlinearity of electrostatic force and the dynamic behaviours of the MVG. The strength of electrostatic force nonlinearity increases with the decrease of nonoverlapping size and the increase of DC bias voltage. It will cause the MVG system to show stiffness softening. Due to the good robustness of the bandwidth, the influence of the electrostatic force nonlinearity on the bandwidth can be neglected. However, the offset of the resonance frequency and amplitude jump caused by the softening characteristics of electrostatic force lead to a decrease in sensitivity and affect stability of sensitivity.

Although the electrostatic nonlinearity has a great influence on the dynamic behaviour, its influence can be avoided by the reasonable design of the comb spacing and DC bias voltage. The effectiveness of linear design of the 
MVG can be guaranteed. There exists a critical value for comb spacing and DC bias voltage. Then, detecting the critical value is particularly important. In this paper, determining the critical values is demonstrated by nonlinear dynamics analysis. The results can be supported by the finite element analysis and numerical simulation.

\section{Data Availability}

The experiment data supporting the analysis were supplied by Tianjin University, and the website where they can be downloaded for free has been cited.

\section{Conflicts of Interest}

The authors declare that there are no conflicts of interest regarding the publication of this paper.

\section{Acknowledgments}

This project was supported by the National Natural Science Foundation of China (Grants nos. 12072234 and 11872044).

\section{References}

[1] P. Greif and B. Boxenhorn, "A vibratory micromechanical gyroscope," AIAA Guidance and Control Conference, vol. 1517, pp. 1033-1040, 1988.

[2] D. Xia, C. Yu, and L. Kong, "The development of micromachined gyroscope structure and circuitry technology," Sensors, vol. 14, no. 1, pp. 1394-1473, 2014.

[3] J. Awrejcewicz, R. Starosta, and G. Sypniewska-Kamińska, "Complexity of resonances exhibited by a nonlinear micromechanical gyroscope: an analytical study," Nonlinear Dynamics, vol. 97, pp. 1819-1836, 2019.

[4] S. H. Jeon, J. Y. Lee, and H. Jung, "Two-mass system with wide bandwidth for SiOG (silicon on glass) vibratory gyroscopes," in Proceedings of the 13th International Conference on SolidState Sensors, Actuators and Microsystems, pp. 539-542, IEEE, Seoul, South Korea, June 2005.

[5] M. Siewe and H. H. Usama, "Homoclinic bifurcation and chaos control in MEMS resonators," Applied Mathematical Modelling, vol. 35, no. 12, pp. 5533-5552, 2011.

[6] M. I. Younis, Mems Linear and Nonlinear Statics and Dynamics, Springer, New York, NY, USA, 2011.

[7] Y. Chang, Q. Zhang, W. Wang, J. Han et al., "Mechanical behaviors of electrostatic microbeams with non ideal supports," Shock and Vibration, vol. 2020, Article ID 4507280, 18 pages, 2020.

[8] N.-C. Tsai and C.-Y. Sue, "Stability and resonance of micromachined gyroscope under nonlinearity effects," Nonlinear Dynamics, vol. 56, no. 4, pp. 369-379, 2009.

[9] E. Kenig, Y. A. Tsarin, and R. Lifshitz, "Homoclinic orbits and chaos in a pair of parametrically driven coupled nonlinear resonators," Physical Review E: Statistical, Nonlinear, and Soft Matter Physics, vol. 84, no. 2, 2011.

[10] E. Tatar, T. Mukherjee, and G. K. Fedder, "Nonlinearity tuning and its effects on the performance of a MEMS gyroscope," in Proceedings of the 2015 Transducers-2015 18th International Conference on Solid-State Sensors, Actuators and Microsystems (TRANSDUCERS), pp. 1133-1136, IEEE, Anchorage, AK, USA, June 2015.
[11] S. A. M. Lajimi, G. R. Heppler, and E. M. Abdel-Rahman, “A parametric study of the nonlinear dynamics and sensitivity of a beam-rigid body microgyroscope," Communications in Nonlinear Science and Numerical Simulation, vol. 50, pp. 180-192, 2017.

[12] S. Y. Hao, H. J. Li, and Q. C. Zhang, "Influence of sense stiffness nonlinearity on the sensitivity stability of a doublesense micro-gyroscope," Journal of Vibration and Shock, vol. 37, no. 24, pp. 63-69, 2018, in Chinese.

[13] M. J. Han, Q. Zhang, H. Hao, and W. Li, "Parametric characteristics and bifurcation analysis of multi-degree-offreedom micro gyroscope with drive stiffness nonlinearity," Micromachines, vol. 10, no. 9, 2019.

[14] W.-M. Zhang, G. Meng, and K.-X. Wei, "Dynamics of nonlinear coupled electrostatic micromechanical resonators under two-frequency parametric and external excitations," Shock and Vibration, vol. 17, no. 6, pp. 759-770, 2010.

[15] B. Chouvion, S. Mcwilliam, and A. A. Popov, "Effect of nonlinear electrostatic forces on the dynamic behaviour of a capacitive ring-based coriolis vibrating gyroscope under severe shock," Mechanical Systems and Signal Processing, vol. 106, pp. 395-412, 2018.

[16] D. D. Liang, X. D. Yang, and W. Zhang, "Linear, nonlinear dynamics, and sensitivity analysis of a vibratory ring gyroscope," Theoretical and Applied Mechanics Letters, vol. 8, no. 6, pp. 393-403, 2018.

[17] S. Sieberer, S. Mcwilliam, and A. A. Popov, "Nonlinear electrostatic effects in MEMS ring-based rate sensors under shock excitation," International Journal of Mechanical Sciences, vol. 157, 2019.

[18] W. Wang, X. Lv, and F. Sun, "Design of micromachined vibratory gyroscope with two degree-of-freedom drive-mode and sense-mode," IEEE Sensors Journal, vol. 12, no. 7, pp. 2460-2464, 2012.

[19] L. Li, J. X. Han, Q. C. Zhang, C. C. Liu, and Z. H. Guo, "Nonlinear dynamics and parameter identification of electrostatically coupled resonators," International Journal of Non-linear Mechanics, vol. 110, pp. 104-114, 2019.

[20] A. H. Nayfeh and D. T. Mook, Nonlinear Oscillations, Wiley, New York, NY, USA, 1979.

[21] L. Xu, L. X. Dong, and W. Wang, "Lateral gap's fringe effect analysis of electrostatic comb structure," Transducer and Microsystem Technologies, vol. 29, no. 8, pp. 26-28, 2010, in Chinese. 